\title{
Regional North American Annual Meeting of the World Federation of Neurology - Research Group on Neuroepidemiology
}

Mount Sinai Medical Center

Miami, Fla., April 15, 2005

\section{Program and Abstracts}

Guest Editors

Ranjan Duara, Miami, Fla.

W.T. Longstreth, Jr., Seattle, Wash.

Stephen K. Van Den Eeden, Oakland, Calif. 


\section{Program}

Abstract No.

\begin{tabular}{|c|c|c|}
\hline $\begin{array}{l}8: 00 \text { AM } \\
8: 45\end{array}$ & $\begin{array}{l}\text { Registration opens } \\
\text { Poster Set-up and Viewing }\end{array}$ & P1-P8 \\
\hline $9: 15$ & Introduction & \\
\hline $9: 35$ & $\begin{array}{l}\text { Epilepsy Genetics in Israeli and Palestinian Arabs } \\
\text { A.D. Korczyn }\end{array}$ & 1 \\
\hline $9: 55$ & $\begin{array}{l}\text { Assessing the Disease Burden due to Epilepsy by Disability Adjusted Life Year in China } \\
\text { D. Ding }\end{array}$ & 2 \\
\hline $10: 15$ & $\begin{array}{l}\text { Prognosis of Status Epilepticus in Adults: Different Therapeutic Management in Two Italian } \\
\text { Areas } \\
\text { R. D`Alessandro }\end{array}$ & 3 \\
\hline $10: 35$ & Break & \\
\hline $11: 05$ & $\begin{array}{l}\text { Idiopathic Parkinson's Disease and Associated Conditions: Results From a Case-Control Study } \\
\text { M. D'Amelio }\end{array}$ & 4 \\
\hline $11: 25$ & $\begin{array}{l}\text { Interaction of Alpha-Synuclein and Tau Genotypes in Parkinson's Disease } \\
\text { D.M. Maraganore }\end{array}$ & 5 \\
\hline $11: 45$ & Business Meeting & \\
\hline 12:15 PM & Lunch & \\
\hline $12: 45$ & Poster Viewing & P1-P8 \\
\hline $1: 15$ & $\begin{array}{l}\text { Keynote Address - 'The Religious Orders Study: Pathologic Mechanisms Linking Risk } \\
\text { Factors to Alzheimer's Disease' } \\
\text { David Bennett, MD }\end{array}$ & \\
\hline $2: 15$ & $\begin{array}{l}\text { Quality of Life and Depression in ALS and MS Patient-Caregiver Couples: A Comparative } \\
\text { Study } \\
\text { A. Chiò }\end{array}$ & 6 \\
\hline $2: 35$ & $\begin{array}{l}\text { Assessing Cognitive Impairment in Persons Living with AIDS in Zambia } \\
\text { M.C. Powell }\end{array}$ & 7 \\
\hline $2: 55$ & $\begin{array}{l}\text { Brain Anatomical Correlates of Gait Variability in High Functioning Older Adults: } \\
\text { A Preliminary Study } \\
\text { C. Rosano }\end{array}$ & 8 \\
\hline
\end{tabular}

$3: 15$

Final Comments and Adjournment 


\section{Oral Presentations}

\section{1 \\ Epilepsy Genetics in Israeli and Palestinian Arabs}

A.D. Korczyn, Z. Afawi, M. Neufeld, S. Kivity, A. Misk,

S. Berkovic

Tel-Aviv, Israel

Objective: To identify novel genetic causes of epilepsy among Arabs in Israel and Palestine. Background: The molecular basis of human epilepsy is slowly being unraveled by analysis of carefully studied families. Populations with large families and access to quality medical care allow the opportunity to accurately define syndromes, determine the clinical genetics of the disorder and eventually the molecular lesion. Methods: Index cases of familial epilepsy were identified in various ways: Search of computerized database of hospitals and outpatient clinics and through personal contacts. Index patients are then contacted by one of us (ZA). After informed consent is obtained, all available family members are interviewed (in spite of political difficulties), a detailed family tree is drawn and blood is taken for DNA extraction and storage. The epilepsy syndrome is being diagnosed by a senior epileptologist based on clinical information, review of old record and investigations and where, appropriate, further EEG and MRI scans. The actual genetic-molecular analysis will be done in Melbourne, Australia. Results: So far 23 families were identified who altogether had 85 cases. It became clear that familial cases of epilepsy are quite common, probably reflecting the common practice of consanguineous marriage and the large number of offspring each couple has.

\section{2 \\ Assessing the Disease Burden Due to Epilepsy by Disability Adjusted Life Year in China}

\section{Ding, Z. Hong, W. Wang, J. Wu, J.W. Sander}

Shanghai and Beijing, China and London, UK

Objective: To demonstrate the application of Disability Adjusted Life Year (DALY) as an aid in health outcome measures to evaluate the epilepsy disease burden in China. To provide Chinese data to achieve a better understanding of disease burden due to epilepsy. Methods: The DALY is the sum of the number of years of survival with disability (Years Lived with Disability, YLD) and the number of years lost due to premature mortality (Years of Live lost, YLL). We calculated the YLD based on the prevalence survey of epilepsy among 66,393 people sampled in 6 provinces in 2000 . The epilepsy mortality data from the Global Burden of Disease study provided the YLL due to epilepsy. We applied sensitivity analysis based on the Chinese literature estimates of mortality range to calculate the plausible range of epilepsy DALY. Results: In 2000, the trend of DALY lost was similar to that of the prevalence of epilepsy in 6 provinces in China. Epilepsy caused 0.73 and
1.29 DALY lost per 1000 population in Shanxi and Ningxia province, which had the lowest and the highest DALY lost among the 6 provinces. The mean DALY lost due to epilepsy was 0.93 per 1,000 population in China. Sensitivity analysis showed that the disease burden due to epilepsy was between 1.37 and 2.31 DALY lost per 1,000 population based on the plausible range of epilepsy mortality. Conclusion: The DALY measure, which includes the extent of disability from epilepsy, provides a useful tool for the epilepsy disease burden assessment.

\section{O 3 \\ Prognosis of Status Epilepticus in Adults: Different Therapeutic Management in Two Italian Areas \\ R. D'Alessandro, L. Vignatelli, R. Rinaldi, C. Tonon, M. Galeotti, P. de Carolis \\ Bologna and Lugo di Romagna, Italy}

Background: The Italian incidence rate of status epilepticus (SE) in adults is similar to that found in other European countries (13.1-16.5/100,000/years vs. 8.0-15.8/100,000/years). However, surveys conducted in two Italian areas in the same region (Bologna and Lugo di Romagna) disclosed a major difference in 30-day case fatality (33 vs. 7\%). Here, we compare the clinical and management variables in the two samples in order to explain this discrepancy. Methods: All the incident adult patients with SE resident in the city of Bologna (1-year observation) and the health district of Lugo di Romagna (2-year observation) were included. The quantitative and qualitative analyses of clinical and therapeutic variables were performed. Results: No major differences were observed in both areas in either the clinical features of SE (etiology, semeiology) or the time of medical management (first observation, first neurological consultancy, EEG exam, first specific therapeutic intervention), whereas the following treatment variables differed: kind of drug after the first two interventions (phenytoin: 29\% in Bologna and $57 \%$ in Lugo di Romagna), management of SE by a neurologist (29\% in Bologna and $79 \%$ in Lugo di Romagna), correct drug treatment (35\% in Bologna and 65\% in Lugo di Romagna), and rate of SE resolution after the first two drug treatments (58\% in Bologna and $85 \%$ in Lugo di Romagna). Conclusions: The two samples examined showed indirect evidence of a correlation between quality of treatment and short-term prognosis of SE.

\section{4 \\ Idiopathic Parkinson's Disease and Associated Conditions: Results from A Case-Control Study}

M. D'Amelio, P. Ragonese, G. Callari, V. Saia, L. Morgante, A. Epifanio, G. Salemi, G. Savettieri

Palermo and Messina, Italy

Objectives: To assess the co-occurrence of multiple diseases, other than cognitive or psychiatric diseases, in patients with Parkinson's disease (PD). Methods: We designed a case-control study 
to investigate the comorbidity in PD patients. PD diagnosis was based on the presence of two out of four cardinal signs in people who were not on anti-parkinsonian therapy, while at least one sign was required in individuals who were taking anti-parkinsonian therapy. Vascular and other forms of Parkinsonism were excluded. PD individuals were matched to PD free individuals $(1: 1)$. Cases and controls were asked for drug use and the occurrence of past and present diseases other than PD. Odds Ratios (OR) were calculated and adjusted for known risk factors. Results: Two-hundred-thirtyseven pairs (54.9\% women) were included in the study. PD patients had a mean age at interview of 65.5 years, mean age at PD onset 59.4 years and mean PD duration 6.3 years. Patients with PD had less disease diagnoses than individuals without $\operatorname{PD}\left(\chi^{2}=33.5512\right.$; $\mathrm{p} \leq 0.0001)$. Multivariate analysis showed a significant inverse association between PD and vascular (OR 0.3; 95\% CI 0.2, 0.5), respiratory (OR $0.2 ; 95 \%$ CI $0.1,0.7$ ), metabolic (OR 0.5; 95\% CI 0.3, 0.9 ), and nervous system diseases (OR $0.4 ; 95 \%$ CI $0.2,1.0$ ). Conclusions: We observed an inverse association between PD and other comorbid disorders. The strict criteria used for idiopathic PD diagnosis might justify some of our findings. For other results further investigations are needed.

\section{5 \\ Interaction of Alpha-Synuclein and Tau Genotypes in Parkinson's Disease}

D.M. Maraganore, C.E. Mamah, T.G. Lesnick, K.J. Strain, M. de Andrade, W.A. Rocca, M.J. Farrer

Rochester, MN, and Jacksonville, FL, USA

Objective: To determine whether common variants in the alpha-synuclein (SNCA) and microtubule-associated protein tau (MAPT) genes interact to confer Parkinson's disease (PD) susceptibility. Background: Aggregation and fibrillization of the SNCA protein may represent key events in the pathogenesis of $\mathrm{PD}$. Mutations in the SNCA gene that promote its aggregation or its fibrillization are causal in families. While these mutations are rare, allelic variability within the SNCA promoter may confer PD susceptibility via a mechanism of gene overexpression and protein aggregation. At low protein concentrations, the fibrillization of SNCA is promoted by the interacting MAPT protein. Haplotype variability within the MAPT gene may confer PD susceptibility by H1-mediated gene over-expression. To date, there are no published studies of possible interactions between common variants of the SNCA and MAPT genes. Methods: We included 557 PD cases and 557 age and gender-matched controls (396 unaffected siblings and 161 unrelated controls). We extracted DNA from blood and genotyped SNCA REP1 alleles and MAPT H1 and H2 haplotypes. We employed a liberalization of the sibling transmission disequilibrium test to assess genetic associations. We focused on five conditional logistic regression interaction models: additive effects, synergistic or antagonistic effects, deferential effects where both are significant but MAPT overrides SNCA, deferential effects where SNCA overrides MAPT, and separate and equal effects where the combined effect of the genes is equal to either alone. We compared the goodness-of-fit of these models using the
Akaike Information Criterion, and assessed their significance using likelihood ratio tests. For the best-fitting interactions model, we calculated odds ratios (OR), 95\% confidence intervals (CI), and p-values. Results: We confirmed the association of SNCA REP1 variability with PD (261/261 bp vs. others; OR, 1.36; 95\% CI, $1.02-1.82 ; \mathrm{p}=0.04)$ and of MAPT variability with $\mathrm{PD}(\mathrm{H} 1 / \mathrm{H} 1$ vs. others; OR, $1.41 ; 95 \% \mathrm{CI}, 1.03-1.93 ; \mathrm{p}=0.03)$. The interaction model that best fit our data revealed an increased risk of PD for persons with either SNCA 261/261 or MAPT H1/H1 genotypes as compared to persons with neither (OR, 1.96; 95\% CI, 1.34-2.86; $p=0.0003$ ), but with separate and equal effects. Conclusions: The SNCA and MAPT gene interactions that we observed are consistent with in vitro experiments that revealed MAPT-mediated fibrillization of SNCA protein at low concentrations (dose threshold effect). We postulate that for persons with genotypes other than SNCA 261/261 (i.e., lower SNCA gene expression levels and SNCA protein concentrations), the MAPT H1/H1 genotype may confer increased PD risk (i.e., higher gene expression levels and MAPT protein-mediated fibrillization of the SNCA protein). However, for persons with the SNCA 261/261 genotype, the MAPT H1/H1 genotype confers no additional effect (because the SNCA protein is already at threshold concentration for self-fibrillization).

\section{O 6 \\ Quality of Life and Depression in ALS and MS Patient-Caregiver Couples: A Comparative Study}

A. Chiò, P. Cavalla, A. Calvo, P. Ghiglione, M. Verzellino,

A. Gauthier, R. Mutani

Turin, Italy

Objective: To assess quality of life (QoL), depression, patients' self-perceived burden and caregivers' burden in ALS and MS patients and their informal caregivers. Methods: This cross-sectional study included $40 \mathrm{MS}$ and 40 ALS patients, paired according to Rankin scale, and their caregivers. Depression and QoL were evaluated with Zung Depression Scale (ZDS) and McGill QoL Questionnaire (MQoL). Clinical status was assessed with EDSS (MS) and ALS-FRS (ALS). Patients' self-perceived burden was measured with the Self-Perceived Burden Scale (SPBS). Caregivers' burden was evaluated with the Caregiver Burden Inventory (CBI). Results: Mean EDSS score was 4.7 (1.9); ALS-FRS score was 25.2 (10.1). Mean MQoL score was 4.8 (1.1) for MS patients, 6.8 (1.0) for MS caregivers, 6.7 (1.6) for ALS patients, and 7.3 (1.1) for ALS caregivers. Mean SPBS score was 84.7 (16.3) for MS, and 71.2 (22) for ALS. Mean CBI score was 68 (13) for MS caregivers, and 50.3 (17.6) for ALS caregivers. Mean ZDS scores were: MS patients, 50.4 (12.4); MS caregivers, 45.9 (11.1); ALS patients, 43.3 (8.7); ALS caregivers, 38.9 (8.1). Twenty-three MS patients (57.5\%), and 19 MS caregivers $(47.5 \%)$ were clinically depressed, versus only 11 (27.5\%) ALS patients and 5 (12.5\%) ALS caregivers. Conclusions: In our series, caregiver burden, depression and QoL scores were worse in MS than in ALS couples. MS patients report a higher selfperceived burden than ALS patients. QoL reported by MS patients 
and caregiver did not correlate with patients' clinical status. The different course of the two diseases may partly explain these differences.

\section{7 \\ Assessing Cognitive Impairment in Persons Living with AIDS in Zambia}

M.C. Powell, D.L. Murman, A. Haworth, E. Velie, G.L. Birbeck

Haslett and East Lansing, MI, USA; Omaha, NE, USA;

Lusaka, Zambia

Objective: Assess cognitive function in persons living with AIDS (PLWAs) in Lusaka, Zambia. Methods: Seventy-six PLWAs were admitted to Kalingalinga during a 3-month enrollment period. Seventeen expired or were transferred to another facility before assessments could be completed. The remaining 59 were screened, 49 met WHO Clinical Case Definition for AIDS, and 48 underwent an adapted mini-mental state exam (MMSE), modified HIV-Dementia scale (MHDS), and Color Trails 1 conducted by non-physician healthcare providers. Students t-tests were used to compare these results with normative data from fifteen healthy subjects with comparable education levels in the same community. Significant cognitive impairment was defined as scoring $\geq 2.5$ SD below the mean of the normal group on any one test, or $<2.5$ and $\geq 1$ SD below the mean on $>2$ tests. Results: PLWAs did not differ from the normative population on age, education, or gender $(\mathrm{p}>0.05)$. Among PLWAs, 53\% reported memory complaints. Twenty (42\%) scored $\geq 2.5$ SDs and $14(29 \%)>1$ SD below the norm on the MMSE. Fifteen PLWAs (32\%) scored $\geq 2.5$ SD and 21 (45\%) were $<2.5$ and $\geq 1$ SD below the norm MHDS score. Thirteen PLWAs (29\%) scored $\geq 2.5$ SD and 9 PLWAs $(20 \%)$ were $<2.5$ and $\geq 1$ SD below the norm on Color Trails 1. Overall, 33 (69\%) PLWAs in this Lusaka-based facility met the study definition for significant cognitive impairment. Conclusions: Cognitive complaints and impairment are common among PLWAs in Zambia. Simple bedside testing conducted by non-physician healthcare providers can identify AIDS-related cognitive impairment in this region.

\section{8 \\ Brain Anatomical Correlates of Gait Variability in High Functioning Older Adults: A Preliminary Study}

C. Rosano, J. Brach, S. Studensky, M.Wu, H. Aizenstein, A.B. Newman

Pittsburgh, PA, USA

Background: Gait variability is a measure of poor gait. It is associated with falls, loss of independence and greater risk of death and hospitalizations. Recent studies in older adults have proposed that attentional control plays an important role in modulating gait coordination and variability but the neural substrates of this association have never been tested. Methods: We studied the associa- tion between MRI measures of brain disease (white matter hyperintensities, subcortical brain infarcts, brain atrophy) and step time variability in 338 cognitively normal older adults ( 78.3 years old, $62 \%$ women, $79 \%$ white). Preliminary volumetric measures of basal ganglia (caudate, putamen, pallidum, thalamus) and of brain areas associated with visuospatial orientation (inferior parietal lobule, Brodmann areas 7, 39, 40) and with mental representation of movements (parahippocampus, precentral gyrus, posterior cingulate cortex, precuneus) were obtained using the Automated Labeling Pathway (ALP) technique. Participants were mostly free from disability ( $94 \%$ had no difficulty performing activities of daily life, $60 \%$ did not report any fall) and prevalence of cardiovascular diseases was less than $20 \%$. Results: Severity of white matter hyperintensities, number of brain infarcts and ventricular enlargement were not associated with step time variability ( $p>0.5$ for all). Smaller volumetric measures of inferior parietal lobule, Brodmann Area 39 and precuneus were significantly associated with greater step time variability, independent of age (partial correlation coefficients: $r^{2}=-0.32$ to $0.42, p<0.003$, after Sidak correction for multiple comparisons). In linear regression models, inferior parietal lobule volume accounted for $22.4 \%$ of the variance of step time variability and was an independent predictor of step time variability, after adjustment for demographics, subclinical cardiovascular disease and total brain volume (fully adjusted model: Standardized $\left.b=-0.046, p=0.02, R^{2}=34.2 \%\right)$. Conclusion: in cognitively normal and high functioning adults atrophy of brain areas associated with visuospatial orientation is associated with greater gait variability.

\section{Poster Presentations}

\author{
P 1 \\ The Diagnosis of Dystonia: A Validity Study \\ E. Beghi, A.M. Bentivoglio D. Fogli, L. Giordano, \\ G. Logroscino, A.M. Papantonio, R. Piolti, G. Rinaldi, \\ P. Simone, L.M. Specchio, P. Tonali, P. Torelli, M. Zarrelli \\ Milano, Roma, San Giovanni Rotondo, Foggia, Monza, and \\ Parma, Italy; Boston, MA, USA
}

Objective: To test the validity of the diagnosis of dystonia. Background: In the absence of biological markers, the diagnosis of dystonia is mostly based on clinical features. Methods: 30 neurologists (12 board-certified and 18 residents) participated in the study (the raters). The sample included 18 patients with cranial (7), cervical (3), upper limb (3), and secondary and/or generalized (5) dystonia, and 11 controls (facial hemispasm 3, tic disorders 3 , chorea 2 , tardive dyskinesia 1 , normal 2). Subjects were followed by one of the authors (the caring neurologist, AMB), chosen as the gold standard. Each rater was asked to examine the video-tape of each subject (including family and personal history data and the neurological examination) and fill a semi-structured questionnaire in- 
cluding the information required to make the diagnosis of dystonia for each given clinical condition. The sensitivity, specificity, and accuracy of the diagnosis were tested by comparing the raters' diagnosis with that of the caring neurologist. The data are expressed as means with 95\% confidence intervals. Results: Dystonia (general) $=$ Sensitivity $0.80(0.76-0.83)$; Specificity $0.72(0.68-0.77)$; Accuracy $0.77(0.74-0.80)$. The corresponding values for boardcertified neurologists were $0.94(0.84-1), 0.64(0.35-0.92)$, and 0.83 $(0.69-0.97)$ and for residents were $0.81(0.77-0.86), 0.67(0.61-$ $0.74)$, and $0.76(0.72-0.80)$. Sensitivity, specificity, and accuracy were slightly higher for cranial dystonia $(0.71,0.65-0.78 ; 0.77$, $074-0.81 ; 0.76,0.73-0.79)$, followed by upper limb dystonia $(0.36$, $0.26-0.46 ; 0.86,0.84-0.89 ; 0.81,0.78-0.84)$, and cervical dystonia (0.76, 0.67-0.85; 0.70, 0.67-0.73; 0.71, 0.68-0.74). Conclusion: The diagnosis of dystonia is fairly accurate, with modest differences between board-certified specialists and residents. The accuracy of the diagnosis is slightly different when cranial, cervical and upper limb dystonia are compared.

\section{P 2 \\ Validity of the Self-Administered GINSEN Form for the Diagnosis of Narcolepsy}

R. D’Alessandro, L. Vignatelli, L. Guidolin, G. Plazzi

Bologna, Italy

Background: The only validated diagnostic tools for the screening of narcolepsy are English and Finnish questionnaires. We assembled an Italian language form (GINSEN form) to develop a validated self-administered questionnaire for the diagnostic screening of narcolepsy. Methods: Five hundred and ninety-nine adults from 17 Italian sleep medicine centres were enrolled. Subjects were selected on the basis of suspicion of four diagnostic groups: 1) narcolepsy; 2) other hypersomniac disorders; 3) neurologic confounding disorders; 4) healthy age-matched people. Patients were classified as having or not having narcolepsy according to international criteria. All subjects self administered the GINSEN form, a collection of Italian validated questionnaires investigating excessive daytime sleepiness (EDS), cataplexy, sleep apnea and insomnia. The best predicting questions were selected from each questionnaire and the diagnostic accuracy measured by discriminant analysis and multivariate logistic regression. Results: Eighteen percent of subjects had narcolepsy. By the discriminant analysis eight questions were found informative for the diagnosis of narcolepsy (4 on EDS, 3 on cataplexy, 1 on sleep apnea); sensitivity was $76 \%$, specificity $93 \%$. By the multivariate logistic regression five questions were selected (the same as above on EDS, cataplexy and sleep apnea, less three); sensitivity was $62 \%$, specificity $97 \%$. Conclusions: Three main items of information are required for the diagnosis of narcolepsy: presence of EDS, cataplexy and absence of sleep apneas. We demonstrated that using clinical data from a self administered form (GINSEN form) the diagnosis could be accurate. Prospective validation of the instrument is now required.

\section{P 3}

\section{Smoking and Tea Consumption Do Not Affect Progression of Parkinson's Disease}

\section{N. Giladi, B. Kandinov, A.D. Korczyn}

Tel-Aviv, Israel

Background: Previous epidemiological studies found a negative association between cigarette smoking and coffee and/or tea drinking with the occurrence of Parkinson's disease (PD). However, it is unknown how these factors affect the rate of progression of the disease. Methods: A retrospective study was conducted among 278 consecutive PD patients. Data on smoking and coffee or tea consumption were obtained through direct or proxy interviews. Cox proportional hazards model was used to estimate whether the dependent variables affect the rate of progression of the disease. Results: Progression of the disease, measured by the time it took to reach Hoehn \& Yahr stage III, was not affected by history of smoking or tea and coffee consumption. Conclusion: Smoking, tea and coffee consumption had no effect on disease progression.

\section{P 4 \\ The Mayo Clinic Cohort Study of Personality and Aging: Design, Sampling and Baseline Results \\ B.R. Grossardt, J.H. Bower, J.E. Ahlskog, K.R. Sanft, M. de Andrade, D.M. Maraganore, W.A. Rocca \\ Rochester, MN, USA}

Objective: To describe the design, sampling, and baseline results of a newly established historical cohort study. Background: From a pre-existing cohort of approximately 50,000 outpatients who completed the Minnesota Multiphasic Personality Inventory (MMPI) at the Mayo Clinic from 1962 through 1965, we established a historical cohort of 7,216 subjects who resided within a 120-mile radius centered in Rochester, Minnesota. Methods: Baseline results for four MMPI scales that have particular relevance to the study of personality and aging were investigated: depression, psychasthenia (anxiety), social introversion, and negativity (pessimism). Trends in scoring were assessed across age- and sex-specific strata. Cohort members are being followed presently to assess age-related outcomes. Results: Baseline raw scores on all four scales were consistently higher for women at all ages. Scores on the depression scale showed an increasing trend with age in both sexes, whereas scores on the psychasthenia (anxiety) scale showed a decreasing trend with age in both sexes. Social introversion and negativity (pessimism) scores remained stable with age. To define abnormal scores (exposure), age- and sex-specific 75th percentile cutoffs of the distribution of raw scores were used. This method was shown to be valid compared to the traditional clinical cut-off ( $\mathrm{T}$ scores plus one standard deviation) by use of receiver operating characteristic curves. Conclusions: This newly established historical cohort study provides opportunities to test hypotheses regarding the link between personality and aging, aging-related diseases, and overall mortality. 


\section{P 5 \\ Long-Term MS Treatment with IFNB-1b: Bringing Cognition Into the Evaluation}

D. Langdon, Y. Lapierre, G. Ebers, P. Kaskel, A. Traboulsee,

S. Hum, C. Wolf, E. Salazar-Grueso, C. Miltenburger

London and Oxford, UK; Montreal and Vancouver, Canada; Berlin, Germany; and Montville, NJ, USA

Background: Cognitive impairment, reported in about 40 $60 \%$ of MS patients, affects both patient management and treatment response. A long-term follow-up trial of the original cohort of the interferon beta-1b (IFNB-1b) pivotal study, a double-blind, placebo-controlled trial to evaluate the safety, tolerability, and efficacy of IFNB-1b in 372 patients with relapsing forms of MS, is now ongoing. Objective: Cognitive evaluation is an objective of this study to investigate the effectiveness of IFNB-1b after more than 15 years of treatment. Methods: A test battery has been selected which is robust in the context of sensory and motor deficits. Pre-morbid IQ was assessed using a reading scale with good psychometric properties (WTAR). Comparisons between pre-morbid optimum intellectual level and current performance allow computing of an index of cognitive deterioration. The cognitive battery includes tests for verbal memory, selective attention, working memory, verbal fluency, and executive skills. French translations have been prepared and validation is in progress. Focusing on the cognitive status of patients in the latter stage of the disease, both in terms of the degree of deterioration and impairment profile, the cognitive data provide a solid basis for future assessment. The relationship of cognitive status to health-related quality of life, anxiety and depression, and employment status are assessed. Cognitive skills are evaluated in the relationship to clinical and MRI parameters. Conclusion: In the context of clinical, MRI and patient-reported outcomes, this study presents a possible methodological solution to evaluate cognition. Furthermore, it will increase our understanding of cognition and related variables under long-term treatment with IFNB-1b.

\section{P 6 \\ Predictive Factors for Ambulation in 150 Stroke Patients Using the Multiple Regression Model}

S. Masiero, R. Avesani, M. Armani, P. Zapparoli, R. Pierobon, V. Postal, M. Ermani

Padova and Verona, Italy

Objective: To investigate the predictive factors for the recovery of ambulation in stroke patients, in the rehabilitation setting. Methods: Side of hemorrhage, type of stroke, hypertension, diabetes, and dyslipidemia were recorded in 150 patients $(80$ males, 70 females). At admission and discharge we performed the Functional Independence Measure (FIM), motor FIM (motFIM), upper and lower Motricity Index (up-MI and low-MI) and Trunk Control Test (TCT). The Functional Ambulation Classification (FAC), assessed at discharge from the rehabilitation setting, was used as the outcome variable. Forward stepwise multiple regression was used, with
FAC as the dependent variable. Results: The up- and low-MI, TCT, FIM and motFIM, and age at entry proved to be significantly linearly related to FAC at discharge, while side of hemorrhage, type of stroke, hypertension, diabetes, dyslipidemia and sex were not. Stepwise multiple regression analysis showed that the independent variables related to FAC were age, TCT and FIM, with a high correlation coefficient $(r=0.82)$. Patients with a higher FAC score had higher values for both TCT and FIM (Kruskal-Wallis, $\mathrm{p}<0.01$ ) and a shorter hospital stay (Kruskal-Wallis, $p=0.004$ ). Conclusions: We used FAC as a measure of outcome in ambulation because of its reliability and sensitivity in stroke patients. Multiple linear regression showed that FAC was explained by TCT, FIM and age, with a high degree of correlation. Interestingly, FAC was not found to be related to side of hemorrhage, type of stroke and hypertension.

\section{P 7 \\ Determinants of Age of Diagnosis of Intracranial Aneurysms}

J. Torner, D. Wiebers, R. Brown, I. Meissner, J. Huston, A. Vasquez

lowa City, IA and Rochester, MN, USA

Background: Intracranial aneurysms are the main cause of subarachnoid hemorrhage (SAH), which accounts for approximately $10 \%$ of strokes. Unlike other forms of stroke the incidence of SAH has not declined in the past decades. SAH occurs at younger ages than other forms of stroke and is strongly predictive of treatment outcome. Methods: Unruptured aneurysm patients were part of the NIH-sponsored International Study of Unruptured Intracranial Aneurysms. In 60 centers 3,077 patients were entered in prospective cohort study of unruptured aneurysms without a history of SAH. The presence, location and characteristics of the intracranial aneurysms were determined by central radiologic review. Factors related to the age were analyzed using univariate contingency table analysis and linear regression. Results: Variables significantly related to age of patients with aneurysms were race, neurological deficits, multiplicity, and aneurysm location. Differential patterns of presenting symptoms related to ischemic disease, seizures, and headaches occurred. Patients age 40-59 consumed more alcohol. Younger patients had a higher frequency of other pathology (PKD, AVM). Older patients had a history of hypertension and were more likely to be former smokers. Age was related to aneurysm size however only after the age of 40 years. Conclusion: Differential factors related to aneurysm formation and presentation may lead to different strategies for screening and evaluation for treatment or observation. 


\section{P 8 \\ Incidence of Multiple Sclerosis (MS) Among First Nations People of Alberta}

S. Warren, L.W. Svenson, K.G. Warren, D. P. Schopflocher,

L. Metz

Edmonton and Calgary, Alberta, Canada

Objective: This study describes the incidence of MS among First Nations people of Alberta, Canada. Background: MS incidence is thought to be low among North American aboriginals based on anecdotal evidence, but no systematic studies have apparently been published. Methods: Physician fee-for-service and hospital records for the years 1994-2002 which mentioned a diagnosis of MS were extracted from Alberta Health Care Insurance Plan data. An individual was considered a case if he/she had had 2 or more physicians visits for MS or 1 or more hospitalizations. First
Nations can be identified since Health Canada pays insurance premiums on their behalf. A case was designated incident if there was no record of services for MS in the previous 5 years. Incidence rates (95\% confidence intervals) were calculated for each year from 1994-2002 per 100,000 population, age-standardized to the 1996 Canadian population. Results: Incidence rates for First Nations were: $1994=12.4 \pm 10.2 ; 1995=7.8 \pm 7.2 ; 1996=7.5 \pm 6.2$; $1997=4.7 \pm 5.0 ; 1998=6.6 \pm 5.3 ; 1999=8.2 \pm 5.5 ; 2000=11.9$ $\pm 7.8 ; 2001=8.4 \pm 6.5 ; 2002=7.6 \pm 5.1$. Incidence rates for the Alberta general population calculated for the same years, in the same way were typically close to three times the First Nations' rates. Conclusions: MS incidence among First Nations is lower than among the general Alberta population, but it is not low by worldwide standards. Although First Nations may have a lesser genetic predisposition to MS, some environmental factor in Alberta may increase their risk. 